\title{
Energy-efficient Optimization for Distributed Opportunistic Scheduling
}

\author{
Andres Garcia-Saavedra, Pablo Serrano, Member, IEEE, Albert Banchs, Senior Member, IEEE
}

\begin{abstract}
Distributed Opportunistic Scheduling (DOS) exploits multiuser diversity in wireless networks without the requirement of a central scheduler. With DOS, users take their own scheduling decisions based on a local observation of the channel to maximize performance. While DOS has been used in the past to optimize throughput-related figures, in this paper we use DOS to optimize the energy performance of the network. We first derive the optimal configuration for the homogeneous scenario, where all devices share the same power consumption behavior, and then propose a heuristic to address the heterogeneous scenario, where devices have different power consumption figures. Simulation results confirm the effectiveness of our approaches.
\end{abstract}

\section{INTRODUCTION}

The key idea of opportunistic scheduling schemes is to exploit the quick quality fluctuations experienced by fading wireless links to dynamically allocate resources to those with best instantaneous conditions [1]-[3]. In this way, rather than aiming at mitigating fading to increase the reliability of the communication, opportunistic mechanisms seek to exploit it to improve overall performance. The recent Distributed Opportunistic Scheduling (DOS) techniques [4] allow each user to take its own scheduling decision based on a local observation of their links to increase the common welfare, without the added complexity of a central controller and the signaling scheme required to provide such entity with timely information about the quality of all the links.

However, most previous works based on DOS focus on optimizing throughput-based utilities (see [5] for a recent survey). In this paper, we use DOS to improve the energy performance of the network. More specifically, the main contributions of this letter are: $(i)$ we formulate the problem of maximizing the energy efficiency in a wireless network with multiple contending nodes using distributed opportunistic techniques, (ii) we derive the optimal ${ }^{1}$ configuration for homogeneous scenarios (i.e., where all stations have the same consumption properties), (iii) we assess the performance of our energyaware DOS configuration, showing notable improvements in efficiency, $(i v)$ we propose a heuristic to configure a heterogeneous scenario, which results very effective while not requiring nodes to exchange information.

A. Garcia-Saavedra is with Hamilton Institute, NUI Maynooth (e-mail: andres.garciasaavedra@nuim.ie).

P. Serrano is with the University Carlos III of Madrid (e-mail: pablo@it.uc3m.es).

A. Banchs is with the University Carlos III of Madrid and the Institute IMDEA Networks (email: banchs@it.uc3m.es).

${ }^{1}$ Throughout the paper, whenever we use the term optimal, we refer to the maximum performance that can be obtained using a distributed opportunistic scheduling technique, where nodes contend for channel access and autonomously decide whether to transmit depending on the channel conditions.

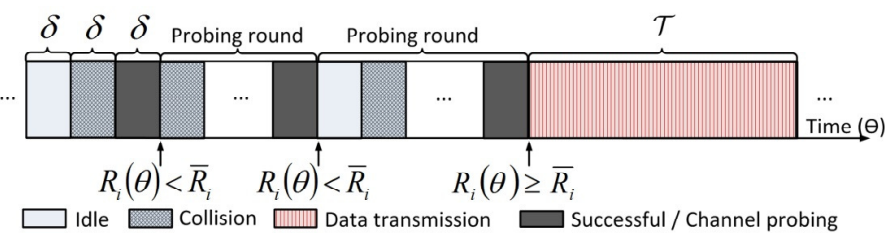

Fig. 1. System model.

To the best of our knowledge, the most related contribution is the work of [6], where authors also use optimal stopping theory to minimize the energy consumption in a wireless network. However, they focus on a point-to-point link (i.e., no interference) and only consider homogeneous scenarios (i.e., only one type of device at a time).

\section{SySTEM MODEL}

Following the same assumptions as in [4], we model DOS as a contention-based single-hop wireless network with $n$ stations, where time is divided into slots of fixed duration $\delta$. At the beginning of each slot, station $i$ contends for channel probing with a given channel access probability, $p_{i}$. A slot can be empty if none of the stations attempt to access the channel. If $n>1$ stations access the channel in the same slot, a collision occurs and the channel is freed for the next slot. There is a successful contention if only one station accesses the channel, which then probes the channel. After this channel probing (which we assume takes only one slot), the station has perfect knowledge of the instantaneous link conditions which can be mapped into a reliable transmission bit rate $R_{i}(\theta)$ at time $\theta$. If the available rate is below a given threshold $\bar{R}_{i}$, station $i$ gives up its transmission opportunity and frees up the channel for re-contention. Otherwise, the station transmits data for a fixed duration of time $\mathcal{T}$, i.e., transmits a frame (see Fig. 1). Finally, we also assume that $R_{i}(\theta)$ remains constant for the duration of a data transmission $\mathcal{T}$ and that different observations of $R_{i}(\theta)$ are independent.

With the above, we have two sets of parameters to tune the performance of the system: the access probabilities $\mathbf{p}=$ $\left\{p_{1}, \ldots, p_{n}\right\}$, and the rate thresholds $\overline{\mathbf{R}}=\left\{\bar{R}_{1}, \ldots, \bar{R}_{n}\right\}$.

\section{A. Throughput Model}

Let us define $l_{i}$ as the average number of bits transmitted by $i$ upon a successful contention, and $T_{i}$ as the average channel holding time. Both $l_{i}$ and $T_{i}$ depend on $\bar{R}_{i}$. Note that when a station wins a contention, it holds the channel for a time $\mathcal{T}+\delta$ if it decides to transmit data, and $\delta$ if it gives up the transmission opportunity. Thus, $T_{i}$ can be computed as

$$
T_{i}=\operatorname{Pr}\left(R_{i}(\theta)<\bar{R}_{i}\right) \delta+\operatorname{Pr}\left(R_{i}(\theta) \geq \bar{R}_{i}\right)(\mathcal{T}+\delta)
$$


If the station decides to use the transmission opportunity, it transmits a number of bits given by $R_{i}(\theta) \mathcal{T}$, which yields

$$
l_{i}=\int_{\bar{R}_{i}}^{\infty} r \mathcal{T} f_{R_{i}}(r) d r
$$

where $f_{R_{i}}(r)$ is the pdf of $R_{i}(\theta)$. Given the above, we can compute the throughput of station $i$ as

$$
r_{i}=p_{s, i} l_{i} / T_{\text {slot }}
$$

where $T_{\text {slot }}=\sum_{j}^{n} p_{s, j} T_{j}+\left(1-p_{s}\right) \delta$ is the average slot length, $p_{s, i}=p_{i} \prod_{j \neq i}\left(1-p_{j}\right)$ is the probability that a mini slot contains a successful contention of station $i$, and $p_{s}=\sum_{i}^{n} p_{s, i}$.

\section{B. Energy Model}

Based on the energy model presented in [7], we characterize the power consumption of station $i$ with three components:

- $\rho_{i}^{i d}$ is the baseline power consumption of the station.

- $\rho_{i}^{t x}$ is the additional power required to transmit a frame.

- $\gamma_{i}^{x g}$ is the energy cost required to build the frame.

Based on the above, we model the average energy consumption $e_{i}$ of station $i$ as the energy consumed over all possible events multiplied by the probability of each event occurring:

$$
e_{i}=p_{e} E_{e, i}+p_{s, i} E_{s, i}+p_{s, \backslash i} E_{s, \backslash i}+p_{c, i} E_{c, i}+p_{c, \backslash i} E_{c, \backslash i}
$$

where

- $p_{e}$ is the probability that an empty slot occurs,

- $p_{s, i}$ is the probability that station $i$ successfully contends to probe the channel,

- $p_{s, \backslash i}$ is the probability that a station different than $i$ successfully contends and probes the channel,

- $p_{c, i}$ is the probability of a collision which station $i$ is involved in,

- $p_{c, \backslash i}$ is the probability of a collision which station $i$ is not involved in;

which can be computed as

$$
\begin{array}{ll}
p_{e}=\prod_{j}\left(1-p_{j}\right), & \\
p_{s, i}=p_{i} \prod_{j \neq i}\left(1-p_{j}\right), & p_{s, \backslash i}=\sum_{j \neq i} p_{j} \prod_{k \neq j}\left(1-p_{k}\right), \\
p_{c, i}=p_{i}-p_{i} \prod_{j \neq i}\left(1-p_{j}\right), & p_{c, \backslash i}=1-p_{i}-p_{e}-p_{s, \backslash i} .
\end{array}
$$

The remaining terms model the energy used on each event: ${ }^{2}$

$$
\begin{aligned}
E_{e, i} & =\rho_{i}^{i d} \delta, \\
E_{s, i} & =\operatorname{Pr}\left(R_{i}(\theta)<\bar{R}_{i}\right)\left((\delta+\mathcal{T})\left(\rho_{i}^{i d}+\rho_{i}^{t x}\right)+\gamma_{i}^{x g}\right)+ \\
& +\operatorname{Pr}\left(R_{i}(\theta) \geq \bar{R}_{i}\right)\left(\rho_{i}^{i d}+\rho_{i}^{t x}\right) \delta, \\
E_{s, \backslash i} & =\sum_{j} \frac{p_{s, j}}{p_{s}}\left(\operatorname{Pr}\left(R_{j}(\theta)<\bar{R}_{j}\right)(\delta+\mathcal{T})\left(\rho_{i}^{i d}\right)+\right. \\
& \left.+\operatorname{Pr}\left(R_{j}(\theta) \geq \bar{R}_{j}\right)\left(\rho_{i}^{i d}\right) \delta\right), \\
E_{c, i} & =\left(\rho_{i}^{i d}+\rho_{i}^{t x}\right) \delta, \quad \quad E_{c, \backslash i}=\rho_{i}^{i d} \delta .
\end{aligned}
$$

${ }^{2}$ Note that, based on the figures reported in [7], we assume that $\rho^{t x}$ does not depend on the transmission rate; and that power adaption is not used, which is a common assumption in distributed opportunistic scheduling works.
Rearranging terms, we can express the average energy consumption of station $i$ as

$$
\begin{aligned}
e_{i} & =\rho_{i}^{i d} \delta+p_{i} \rho_{i}^{t x} \delta+ \\
& +\sum_{j \neq i} p_{s, j} \operatorname{Pr}\left(R_{j}(\theta) \geq \bar{R}_{j}\right)\left(\rho_{i}^{i d} \mathcal{T}\right)+ \\
& +p_{s, i} \operatorname{Pr}\left(R_{i}(\theta) \geq \bar{R}_{i}\right)\left(\mathcal{T}\left(\rho_{i}^{i d}+\rho_{i}^{t x}\right)+\gamma_{i}^{x g}\right) .
\end{aligned}
$$

Finally, we can model the average power consumption of station $i$ as the expected energy consumed over the average slot length, i.e.,

$$
\pi_{i}=e_{i} / T_{\text {slot }}
$$

\section{Optimal CONFIGURATION}

Our aim is to design a configuration strategy to improve the energy performance of a network using DOS. More specifically, our performance optimization criteria will be based on the energy efficiency of station $i\left(\eta_{i}\right)$, defined as the ratio of transmitted information over the energy consumed, i.e.,

$$
\eta_{i}=p_{s, i} l_{i} / e_{i}
$$

\section{A. Homogeneous Scenario}

We first consider the homogeneous case, where all the stations have the same energy profile and channel conditions. In this scenario, the tunable set of parameters of the system is reduced to just two, i.e., the transmission probability $p$ and the rate threshold $\bar{R}$ used by all stations, and our aim is to optimize the overall energy efficiency of the network. ${ }^{3}$

Following [8], we set $p=1 / n$, as this configuration gives an optimal success probability. Based on this, the remaining challenge is to find the threshold policy that maximizes overall energy efficiency and, to this aim, we use optimal stopping theory [9]. ${ }^{4}$ If we consider each successful contention of a station as a "round", the challenge is to compute in which round and under which conditions the station should transmit after accessing (i.e., probing) the channel to maximize performance. We refer to stopping rule $N \in \mathcal{N}$ to the strategy of deciding to transmit at channel probing round $N$ out of the set of possible choices $\mathcal{N}$. The problem can be formulated as a maximum rate of return problem, where we invest in energy during the probing rounds $(\psi)$ and expect a payoff in data delivered $(Y)$. To maximize the energy efficiency, the optimization problem results

$$
\max _{N \in \mathcal{N}} \frac{E\left[Y_{N}\right]}{E\left[\psi_{N}\right]}=\max _{N \in \mathcal{N}} \frac{E\left[R_{N} \mathcal{T}\right]}{E\left[\sum_{k=1}^{N} e_{c p, k}+\gamma_{x g}+\mathcal{T} \pi_{t x}\right]}=\lambda
$$

where $R_{N}$ is the observed available transmission bit rate at round $N, e_{c p, k}$ is the energy consumed in round $k$, and $\gamma_{x g}+\mathcal{T} \pi_{t x}$ is the energy consumed during a frame transmission, with $\pi_{t x}=\rho_{t x}+n \rho_{i d}$. The rate of return, $\lambda$, is the expected energy efficiency (in bits/joule) rewarded for using such strategy.

\footnotetext{
${ }^{3}$ Given that all stations have the same power properties, the configuration that maximizes energy efficiency is the same for all, and fair. For readability, we remove the subindex $i$ of the power consumption figures.

${ }^{4}$ Optimal stopping is the technique used in [4] to maximize throughput performance using DOS.
} 
Following [9], we transform the problem defined in (10) into an ordinary stopping rule problem, i.e.,

$\max E\left[Y_{N}-\lambda \psi_{N}\right]=\max E\left[R_{N} \mathcal{T}-\left(\sum_{k=1}^{N} e_{c p, k}+\gamma_{x g}+\mathcal{T} \pi_{t x}\right)\right]$

where we invest $c_{N}=\sum_{k=1}^{N} \lambda e_{c p, k}$ to receive $X_{N}=$ $R_{N} \mathcal{T}-\lambda\left(\gamma_{x g}+\mathcal{T} \pi_{t x}\right)$ when we decide to transmit. Our objective is to find the $\lambda$ and the stopping rule $N$ such that the optimal expected reward of this dual problem is zero, i.e., $V^{*} \equiv \sup _{N^{*} \in \mathcal{N}} E\left[Y_{N}-\lambda \psi_{N}\right]=0$, because then, according to Th.1 in Ch.6 of [9], $N$ will be the optimal rule for our original problem (10) and $\lambda$ will be the maximum rate of return, i.e., the maximum energy efficiency of the network.

In these circumstances, it can be shown that the optimal stopping rule is

$$
N^{*}=\min \left\{N \geq 1: X_{N} \geq V^{*}\right\}
$$

and that $V^{*}$ satisfies the optimality equation:

$$
V^{*}=E\left[\max \left\{X_{1}, V^{*}\right\}\right]-c_{1} .
$$

Given that $V^{*}\left(\lambda^{*}\right)=0$ and that $R_{N}$ has the same distribution for all $N$, the above results in

$$
E\left[\max \left\{R \mathcal{T}-\lambda^{*}\left(\bar{e}_{c p}+\gamma_{x g}+\mathcal{T} \pi_{t x}\right),-\lambda^{*} \bar{e}_{c p}\right\}\right]=0
$$

where $\bar{e}_{c p}$ is the expected energy consumed during a round,

$\bar{e}_{c p}=\sum_{i}^{n} E_{c, i} K_{c, i}+\sum_{i}^{n} E_{c, i} K_{c, i}+\sum_{i}^{n} E_{e, i} K_{e}+\delta\left(\sum_{i}^{n} \rho_{i d}+\rho_{t x}\right)$

with $K_{c, i}, K_{c, \backslash i}$ and $K_{e}$ being the average fraction of time with collisions involving station $i$, collisions not involving $i$ and empty slots, respectively, in one probing round. We can estimate these averages as

$$
K_{c, i}=\frac{\left(\frac{1}{p_{s}}-1\right) p_{c, i}}{1-p_{s}}, K_{c, \backslash i}=\frac{\left(\frac{1}{p_{s}}-1\right) p_{c, \backslash i}}{1-p_{s}}, K_{e}=\frac{\left(\frac{1}{p_{s}}-1\right) p_{e}}{1-p_{s}} .
$$

Equation (13) can be rewritten as

$$
\lambda^{*} \bar{e}_{c p}=E\left[R \mathcal{T}-\lambda^{*}\left(\gamma_{x g}+\mathcal{T} \pi_{t x}\right)\right]^{+},
$$

which is a fixed point equation that can be solved by each station with numerical techniques using local information only.

The DOS protocol we are optimizing, however, requires the following threshold-based rule:

$$
N=\min \left\{N \geq 1: R_{N} \geq \bar{R}^{*}\right\},
$$

which can be derived from (11) by defining the transmission rate threshold as $\bar{R} \equiv \lambda \frac{\gamma_{x g}+\mathcal{T} \pi_{t x}}{\mathcal{T}}$. Its optimal value $\bar{R}^{*}$ then can be found by solving

$$
\bar{R}^{*} \frac{\bar{e}_{c p}}{\left(\gamma_{x g}+\mathcal{T} \pi_{t x}\right)}=E\left[R-\bar{R}^{*}\right]^{+} .
$$

\section{B. Heterogeneous Scenario}

In a heterogeneous scenario, where stations have different energy consumption profiles, optimizing the overall energy efficiency results in a very unfair allocation of resources [10], i.e., all throughput is given to one set of stations. To address this, [10] proposes the Energy-efficiency Fair (EF) criterion, which maximizes the utility function $E F \equiv \sum_{i} \log \left(\eta_{i}\right)$. This objective function aims at a high overall throughput allocation (by aggregating individual utilities) while preventing the starvation of any station (penalizing null allocations of $\eta_{i}$ ).

Based on the above, we formulate our optimization as

$$
\underset{\mathbf{p}, \overline{\mathbf{R}}}{\operatorname{maximize}} \sum \log \left(\eta_{i}\right)
$$

Deriving the optimal configuration based on the above formulation, though, results too complex due to the non-linear relationship among the decision variables $\mathbf{p}=\left\{p_{1}, \ldots, p_{n}\right\}$ and $\overline{\mathbf{R}}=\left\{\bar{R}_{1}, \ldots, \bar{R}_{n}\right\}$ and the objective function. To address this, we propose a heuristic to configure the network. First, similarly to the homogeneous case, all stations configure the same access probability $p_{i}=1 / n$, which grants fairness in terms of channel access probabilities. The remaining challenge is the configuration of the $\bar{R}_{i}$ 's to maximize (19), a criterion that favors energy-efficient stations while preventing starvation (due to the properties of the logarithm). To this aim, each device $i$ configures its own threshold, following (18), assuming that all stations have $i$ 's power profile (like in the homogeneous case), which results in a larger threshold for energy-hungry stations but without extreme divergences in their configuration. In this way, a station only needs to know its power consumption behavior and the number of contending stations $n$, which can be easily inferred by sniffing the wireless channel [11]. The effectiveness of this approach is assessed in the next section.

\section{Performance Evaluation}

We evaluate the performance of our strategies by means of simulations. We use an event-driven simulator that models the channel after Shannon channel capacity: $R(h)=W \log _{2}(1+$ $S N R|h|^{2}$ ) bits/s, where $W$ is the channel bandwidth, $S N R$ is the normalized average Signal to Noise Ratio and $h$ is the random gain of Rayleigh fading. For space reasons, we limit ourselves to the case of $S N R=6 \mathrm{~dB}$ and $W=10 \mathrm{MHz}$, but we tested scenarios with different conditions and the performance gains were relatively similar. Moreover, we configure DOS with $\mathcal{T} / \delta=10$ and we model the power consumption profile of the nodes after the three devices characterized in [7], namely "Soekris", "Alix" and "Linksys".

\section{A. Homogeneous Scenario}

The first scenarios we evaluate are populated with homogeneous devices, that is, all the stations have the same power profiles. We set up three different scenarios with the three considered devices. For each scenario, we vary the total number of stations and compare the overall energy efficiency of our configuration ("Green DOS") against a configuration that maximizes overall throughput ("DOS") and a configuration that tunes the access probabilities to maximize overall 

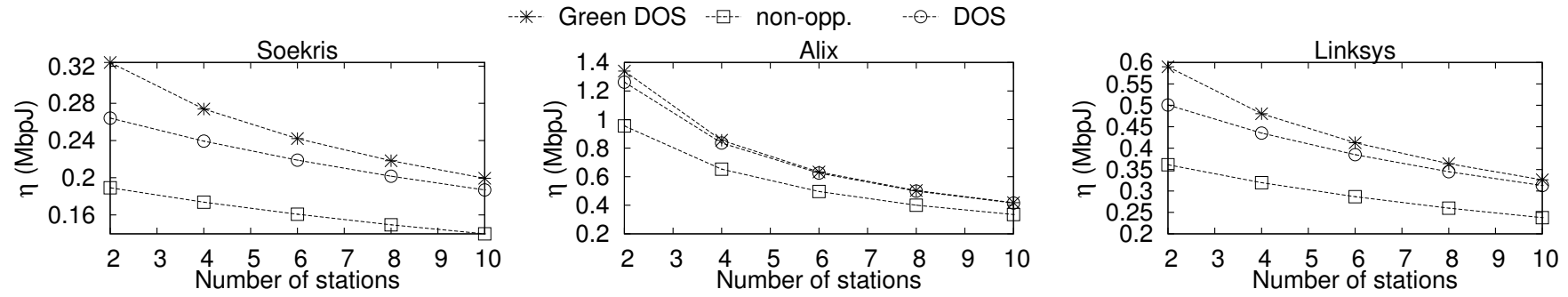

Fig. 2. Energy efficiency in a homogeneous scenario.

throughput and the rate threshold to zero, i.e., it does not use opportunistic access (“non-opp."). The results, depicted in Fig. 2, show that our strategy increases the energy efficiency with respect to DOS up to $30 \%$ for the case of the "Soekris" and up to $12 \%$ for the "Alix", the most energy-efficient device. In all cases, our strategy performs much better than the nonopportunistic configuration, with an improvement that ranges between $30 \%$ and $85 \%$. We also note that $\eta$ decreases for all strategies as the number of stations $n$ increases: this is because the total throughput remains approx. constant with $n$, while the power consumption grows with $n$.

\section{B. Heterogeneous Scenario}

We set up now two heterogeneous scenarios with $n=10$ stations, five of them modeled after the "Soekris" device. The remaining five stations are modeled after the "Alix" firstly, and after the "Linksys" secondly. We then compare, in Fig. 3, the throughput performance of each group of stations and the overall energy efficiency for the same configuration strategies as before. To evaluate how close our heuristic strategy is from the EF-optimal configuration, we solve using numerical techniques the optimization problem described in (19) with complete knowledge of the network, and include its outcome in Fig. 3 (labeled as "Optimal"). Note that this last strategy mimics an omniscient centralized scheduler with zero communication delays using the same access protocol.

The conclusion from these experiments is twofold. First, the heuristic strategy proposed in Section III-B follows very closely the performance of the optimal configuration, with differences in performance below 5\%. Second, the EF criterion provides a good trade-off between maximizing overall energy efficiency and maintaining throughput fairness among the stations. The overall energy efficiency improves by approx. $15 \%$ with respect to the throughput optimization in both scenarios, and above 50\% comparing to the "non-opportunistic"

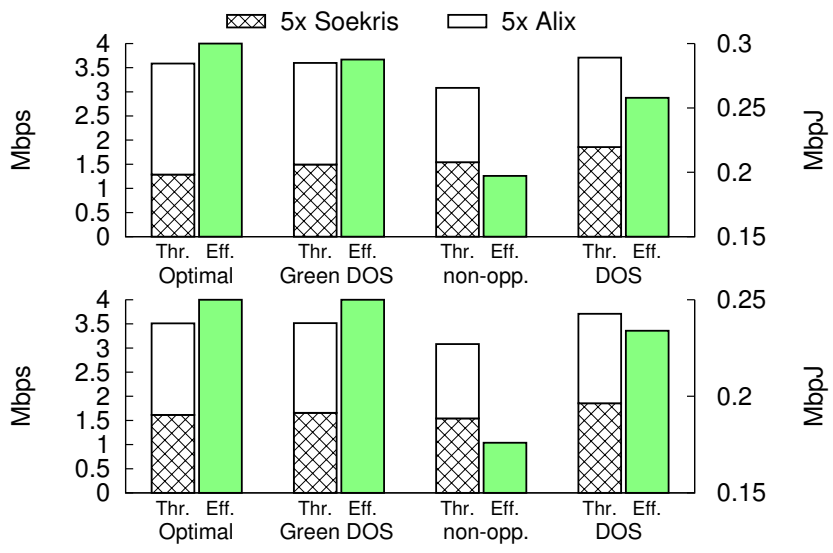

Fig. 3. Energy efficiency in heterogeneous scenarios. configuration, while the degree of fairness has been kept high, with a Jain's index [12] of approx. 0.90 in both cases.

\section{CONCLUSIONS}

We have analyzed the use of DOS techniques to address energy efficiency in wireless networks. Building on a recent model for the energy consumption of wireless stations, we have derived the optimal threshold policy for homogeneous scenarios, where all stations have the same power consumption profile. For the case of heterogeneous scenarios, we have proposed a heuristic to improve energy performance while guaranteeing some fairness among stations. Simulation results confirm that our schemes are able to significantly improve the energy efficiency of wireless networks.

\section{ACKNOWLEDGMENTS}

This work has been partly supported by the European Community through the CROWD project (FP7-ICT-318115).

\section{REFERENCES}

[1] R. Knopp and P. A. Humblet, "Information capacity and power control in single-cell multiuser communications," in Proc. of IEEE International Conference on Communications (ICC), 1995, pp. 331-335.

[2] X. Liu, E. K. P. Chong, and N. B. Shroff, "Opportunistic transmission scheduling with resource-sharing constraints in wireless networks," IEEE Journal on Selected Areas in Communications, vol. 19, no. 10, pp. 2053-2064, 2001.

[3] N. Sharma and L. H. Ozarow, "A study of opportunism for multipleantenna systems," IEEE Trans. on Information Theory, vol. 51, no. 5, pp. 1804-1814, 2005.

[4] D. Zheng, W. Ge, and J. Zhang, "Distributed opportunistic scheduling for ad hoc networks with random access: an optimal stopping approach," IEEE Transactions on Information Theory, vol. 55, no. 1, January 2009.

[5] A. Asadi and V. Mancuso, "A survey on opportunistic scheduling in wireless communications," IEEE Surveys and Tutorials on Communications, no. 99, pp. 1-18, 2013.

[6] M. Poulakis, A. Panagopoulos, and P. Constantinou, "Channel-aware opportunistic transmission scheduling for energy-efficient wireless links," Vehicular Technology, IEEE Transactions on, vol. 62, no. 1, pp. 192204, 2013.

[7] A. Garcia-Saavedra, P. Serrano, A. Banchs, and G. Bianchi, "Energy consumption anatomy of 802.11 devices and its implication on modeling and design," in Proc. of the 8th international conference on Emerging networking experiments and technologies. ACM, 2012, pp. 169-180.

[8] P. Gupta, Y. Sankarasubramaniam, and A. Stolyar, "Random-access scheduling with service differentiation in wireless networks," in Proceedings of IEEE INFOCOM, Miami, FL, March 2005.

[9] T. S. Ferguson. Optimal stopping and applications. [Online]. Available: http://www.math.ucla.edu/ tom/Stopping/Contents.html

[10] A. Garcia-Saavedra, P. Serrano, A. Banchs, and M. Hollick, "Balancing energy efficiency and throughput fairness in IEEE 802.11 WLANs," Pervasive and Mobile Computing, 2012.

[11] G. Bianchi and I. Tinnirello, "Kalman filter estimation of the number of competing terminals in an ieee 802.11 network," in INFOCOM 2003, vol. 2, March 2003, pp. 844-852 vol.2.

[12] R. Jain, D. M. Chiu, and W. Hawe, "A quantitative measure of fairness and discrimination for resource allocation in shared computer systems," DEC, Tech. Rep. TR-301, 1984. 